\title{
Гетероструктура 2D SiC/Si: электронные состояния и адсорбционная способность
}

\author{
(С) С.Ю. Давыдов ${ }^{1}$, А.В. Зубов ${ }^{2}$ \\ ${ }^{1}$ Физико-технический институт им. А.Ф. Иоффе Российской академии наук, \\ 194021 Санкт-Петербург, Россия \\ ${ }^{2}$ Санкт-Петербургский национальный исследовательский университет \\ информационных технологий, механики и оптики (ИТМО), \\ 197101 Санкт-Петербург, Россия \\ E-mail: Sergei_Davydov@mail.ru \\ Поступила в Редакцию 20 января 2020 г. \\ В окончательной редакции 20 февраля 2020 г. \\ Принята к публикации 20 февраля 2020 г.
}

Предложена модель гетероструктуры, представляющей собой монослой карбида кремния, сформированный на массивной кремниевой подложке. Рассмотрена задача об адсорбции атомов щелочных металлов и галогенов на атомах углерода и кремния поверхности гетероструктуры 2D SiC/Si. Приведены аналитические оценки перехода заряда и энергии адсорбции.

Ключевые слова: двумерные гексагональные слои, полупроводниковая подложка, плотность состояний, адсорбция.

DOI: $10.21883 /$ FTP.2020.07.49507.9350

\section{1. Введение}

В постграфеновый период начался активный (главным образом теоретический) поиск новых двумерных (2D) материалов [1-8]. Такие материалы отличаются от графена наличием щели в спектре электронных состояний, что делает их более удобными для приборного использования. Среди этих материалов следует выделить $2 \mathrm{D}$ соединения $\mathrm{A}_{N} \mathrm{~B}_{8-N}[3,4,8]$, учитывая особое место их $3 \mathrm{D}$ прародителей в полупроводниковой электронике. Большой интерес, на наш взгляд, представляет двумерный карбид кремния (2D $\mathrm{SiC})$. Во-первых, 2D $\mathrm{SiC}$, как и 3D $\mathrm{SiC}$, должен быть устойчив к механическим, тепловым и радиационным воздействиям. Во-вторых, по ряду данных, 2D $\mathrm{SiC}$, в отличие от $3 \mathrm{D} \mathrm{SiC}$, является прямозонным полупроводником (см. подборку данных в [9]), что позволяет использовать этот материал в оптоэлектронике наравне с нитридами галлия и алюминия.

Для того чтобы понять, как 2D материал работает в приборных структурах, необходимо учитывать влияние подложки, рассматривая, таким образом, не свободную, а эпитаксиальную структуру. В этом плане представляют интерес работы [10-12], где исследовалась гетероструктура, образованная нанослоем карбида кремния, сформированного на кремниевой подложке $(\mathrm{SiC} / \mathrm{Si})$. Эта гетероструктура использовалась как платформа для роста ряда различных нанопленок. Не следует забывать также роль $\mathrm{SiC}$-подложки в формировании слоев графена.

B настоящей работе мы рассмотрим систему 2D $\mathrm{SiC} / \mathrm{Si}$. Воспользовавшись результатами работ [13-15], мы определим плотность электронных состояний такой гетероструктуры и изучим ее адсорбционные свойства.
Напомним, что адсорбция является элементарным актом многих поверхностных процессов.

\section{2. Плотность состояний гетероструктуры 2D SiC/Si}

\section{1. Общие соотношения}

Модельный подход к описанию эпитаксиальных графеноподобных соединений $\mathrm{A}_{N} \mathrm{~B}_{8-N}$ (ГПС) представлен В [13-15]. В низкоэнергетическом приближении, когда электронный спектр можно линеаризовать, плотность состояний плоского слоя эпитаксиального ГПС имеет вид

$$
\begin{gathered}
\rho_{\mathrm{AB}}(\widetilde{\Omega})=\frac{I_{\mathrm{AB}}(\widetilde{\Omega})}{\pi \sqrt{3} t}, \\
I_{\mathrm{AB}}(\widetilde{\Omega})=\frac{\Gamma(\omega)}{2 \pi t} \ln \frac{\left|\xi^{4}+b \xi^{2}+c\right|}{c} \\
+\frac{\widetilde{\Omega}}{\pi t}\left(\operatorname{arctg} \frac{2 \xi^{2}+b}{4 \Gamma(\omega) \widetilde{\Omega}}-\operatorname{arctg} \frac{b}{4 \Gamma(\omega) \widetilde{\Omega}}\right) .
\end{gathered}
$$

Здесь $\widetilde{\Omega}=\omega-\varepsilon(\omega), \quad b=-2\left(\widetilde{\Omega}^{2}-\Delta^{2}-\Gamma^{2}(\omega)\right), \omega-$ энергетическая переменная, $c=\left(\widetilde{\Omega}^{2}-\Delta^{2}\right)^{2}+\Gamma^{2}(\omega)$ $\times\left[\Gamma^{2}(\omega)+2 \Delta^{2}+2 \widetilde{\Omega}^{2}\right], \quad \varepsilon(\omega)=\bar{\varepsilon}+\Lambda(\omega), \bar{\varepsilon}=\left(\varepsilon_{a}+\varepsilon_{b}\right) / 2$, $\varepsilon_{a(b)}$ - энергия p-орбитали атома $\mathrm{A}(\mathrm{B}), \Delta=$ $=\left|\varepsilon_{a}-\varepsilon_{b}\right| / 2$ - полуширина запрещенной зоны ГПС (щели), $\Lambda(\omega)$ и $\Gamma(\omega)$ - функции сдвига и уширения квазиуровней атомов А и $\mathrm{B}, t-$ энергия перехода электрона между $p$-орбиталями ближайших соседей, $\xi=t \sqrt{2 \pi \sqrt{3}} \approx 3.3 t$. В области запрещенной зоны 
кремния, где $\Gamma(\omega)=0$, плотность состояний ГПС есть

$$
\rho_{\mathrm{AB}}(\widetilde{\Omega})=\left\{\begin{array}{l}
\frac{|\widetilde{\Omega}|}{\pi \sqrt{3} t}, \Delta \leq|\widetilde{\Omega}| \leq R, \\
0,|\widetilde{\Omega}|<\Delta,|\widetilde{\Omega}|>R
\end{array}\right.
$$

где $R=\sqrt{\xi^{2}+\Delta^{2}}$. Это выражение справедливо и для плотности состояний $\rho_{\mathrm{AB}}^{0}(\omega)$ свободных ГПС при замене $\widetilde{\Omega}=\omega-\varepsilon(\omega)$ на $\Omega=\omega-\bar{\varepsilon}$.

Для плотности состояний подложки $\rho_{\text {sub }}(\omega)$ выберем параболическую модель [16]:

$$
\begin{aligned}
& \rho_{\text {sub }}(\omega)= \\
& =B\left\{\begin{array}{l}
\sqrt{2 \omega / E_{g}-1}, \quad 1<2 \omega / E_{g} \leq 2 W / E_{g}+1 ; \\
\sqrt{-2 \omega / E_{g}-1}, \quad-1-2 W / E_{g} \leq 2 \omega / E_{g}<-1 ; \\
0, \quad\left|2 \omega / E_{g}\right| \leq 1, \quad\left|2 \omega / E_{g}\right|>1+2 W / E_{g} \mid,
\end{array}\right.
\end{aligned}
$$

где $E_{g}$ - ширина запрещенной зоны подложки, в центр которой помещено начало отсчета энергии, $W-$ ширина зон разрешенных состояний, $B=A \sqrt{E_{g} / 2}-$ коэффициент с размерностью ${ }^{-1}$. Функция уширения $\Gamma(\omega)=\pi V^{2} \rho_{\mathrm{AB}}(\omega)$, где $V-$ матричный элемент взаимодействия ГПС с подложкой. Функция сдвига, по определению равная

$$
\Lambda(\omega)=\frac{1}{\pi} P \int_{-\infty}^{\infty} \frac{\Gamma\left(\omega^{\prime}\right) d \omega^{\prime}}{\omega-\omega^{\prime}} d \omega^{\prime}
$$

где $P-$ символ главного значения, имеет вид:

$$
\Lambda(\omega)=C \begin{cases}f_{-}(\omega)-l_{-}(\omega), & 2 \omega / E_{g}<-1, \\ f_{-}(\omega)-f_{+}(\omega), & -1 \leq 2 \omega / E_{g} \leq 1, \\ -f_{+}(\omega)+l_{+}(\omega), & 2 \omega / E_{g}>1,\end{cases}
$$

где $C=\pi B V^{2}$ и

$$
\begin{gathered}
f_{ \pm}(\omega)=2 \sqrt{1 \pm 2 \omega / E_{g}} \arctan \frac{\sqrt{2 W / E_{g}}}{\sqrt{1 \pm 2 \omega / E_{g}}}, \\
l_{ \pm}(\omega)=\sqrt{-1 \pm 2 \omega / E_{g}} \ln \mid \frac{\sqrt{-1 \pm 2 \omega / E_{g}}+\sqrt{2 W / E_{g}}}{\sqrt{-1 \pm 2 \omega / E_{g}}-\sqrt{2 W / E_{g}}} .
\end{gathered}
$$

\section{2. Гетероструктура $2 \mathrm{D} \mathrm{SiC/Si}$}

Начнем с оценки параметров задачи. Для кремния ширина запрещенной зоны $E_{g}=1.11$ эВ и электронное сродство $\chi=3.99$ эВ [17]. Для массивного карбида кремния значения $E_{g}$ для $3 C, 6 H$ и $4 H$ политипов равны соответственно $2.40,3$ и 3.23 эВ, для электронного сродства в том же ряду имеем $4,3.45$ и 3.17 эВ [18]. Отсюда следует, что центры запрещенных зон для $3 C, 6 H$ и $4 H$ политипов отвечают соответственно энергиям $E_{0} \approx-0.66,-0.41$ и -0.24 эВ. Гетеропереходы $6 H-\mathrm{SiC} / \mathrm{Si}$ и $4 H-\mathrm{SiC} / \mathrm{Si}$ являются охватывающими (straddling) переходами I рода, тогда как гетеропереход $3 C$ - SiC/Si представляет собой, строго говоря, ступенчатый (staggered) переход II рода, хотя значения электронного сродства компонентов этого перехода практически совпадают.

Двумерный гексагональный карбид кремния рассматривался нами в работе [9], где для ширины щели $2 \Delta$ были получены значения 2.45 и 3.48 эВ при использовании таблиц атомных термов Хермана-Скиллмана [19] и Манна [20] соответственно. Там же отмечено, что, согласно имеющимся численным расчетам из первых принципов, значения $2 \Delta$ лежат в интервале 2.34-4.19эВ [9]. Матричный элемент $\pi$-связи $p$-орбиталей ближайших $\mathrm{C}$ и $\mathrm{Si}$ атомов, рассчитанный по методу связывающих орбиталей Харрисона [21], равен $t=1.53$ эВ. Для дальнейшего рассмотрения примем $\Delta=t$. Множитель $A$ определяется из условия нормировки $A W^{3 / 2}=6$ (четыре электрона на зону). Так как для кремния $W \approx 12$ эВ [22], имеем $A \sim 0.14$ э $^{-3 / 2}$ и $B \approx 0.11$ э $\mathrm{B}^{-1}$. Положив $V=t$, получим $C=0.52$ эВ. Ширина зон разрешенных состояний $2 \mathrm{D} \mathrm{SiC} \mathrm{равна} w=R-\Delta \approx 2.45 t, R \approx 3.45 t$. Величину $\bar{\varepsilon}$ определить трудно, поэтому будем рассматривать $\bar{\varepsilon}$ как параметр. Введем безразмерные величины, приняв за единицу энергии $t$; приведенные плотности состояний равны $\rho_{\mathrm{SiC}}^{*}=\widetilde{\rho}_{\mathrm{SiC}} \cdot \pi t \sqrt{3}=I_{\mathrm{SiC}}, \rho_{\mathrm{Si}}^{*}=\rho_{\mathrm{Si}} \cdot \pi t \sqrt{3}$, где $\rho_{\mathrm{Si}}=\rho_{\text {sub }}($ cм. (3)).

Графики $I_{\mathrm{SiC}}, I_{\mathrm{SiC}}^{0}=\rho_{\mathrm{SiC}}^{0} \cdot \pi t \sqrt{3}$ и $\rho_{\mathrm{Si}}^{*}$ при $\bar{\varepsilon}=0$ представлены на рис. $1, a$, на рис. $1, b$ приведен график функции сдвига $\Lambda(\omega)$. Рассмотрим область запрещенной зоны кремниевой подложки. Варьируя значение $\bar{\varepsilon}$, будем отслеживать, как при этом изменяется приведенная плотность состояний $I_{\mathrm{SiC}}(\omega)$. Начнем с области энергий $|\omega| \leq E_{g} / 2$. При $\bar{\varepsilon}=0$ запрещенная зона гетероструктуры 2D SiC/Si пуста (так как $E_{g} / 2<\Delta=t$, запрещенная зона $2 \mathrm{D} \mathrm{SiC/Si} \mathrm{совпадает} \mathrm{с} \mathrm{запрещенной}$ зоной кремния). Теперь начинаем смещать $\bar{\varepsilon}$ в сторону отрицательных энергий и отыскивать решения $\varepsilon_{C}$ уравнения $\bar{\varepsilon}=\varepsilon_{C}-\Lambda\left(\varepsilon_{C}\right)-\Delta$, отвечающие энергии дна зоны проводимости 2D SiC. Результаты представлены на pис. 2. При $\bar{\varepsilon} \approx 0.26 t$ (на рис. 2, $a$ это значение отмечено правым темным кружком на оси абсцисс) дно зоны проводимости 2D $\mathrm{SiC}$ совмещается с верхним краем запрещенной зоны Si-подложки: $\varepsilon_{C} \approx 0.36 t=E_{g} / 2$. При $\bar{\varepsilon}=-t$ получаем $\varepsilon_{C}=0$ : половина запрещенной зоны кремния перекрывается плотностью состояний $2 \mathrm{D} \mathrm{SiC}$. При дальнейшем смещении $\varepsilon_{C}$ вниз по шкале энергии плотность состояний $2 \mathrm{D} \mathrm{SiC}$ продвигается в левую половину запрещенной зоны кремния и при $\bar{\varepsilon}_{C} \approx-1.74 t$ (на рис. 2, $a$ это значение отмечено левым темным кружком на оси абсцисс) имеем $\varepsilon_{C} \approx-0.36 t=-E_{g} / 2$, т.е. край зоны проводимости $2 \mathrm{D} \mathrm{SiC}$ совмещается с потолком валентной зоны подложки. В этом случае система 2D SiC/Si, которая до этого была полупроводниковой, становится металлической и остается таковой до $\bar{\varepsilon} \approx-2.71 t$, когда потолок зоны проводимости 2D $\mathrm{SiC}$ совмещается с дном запрещенной зоны 

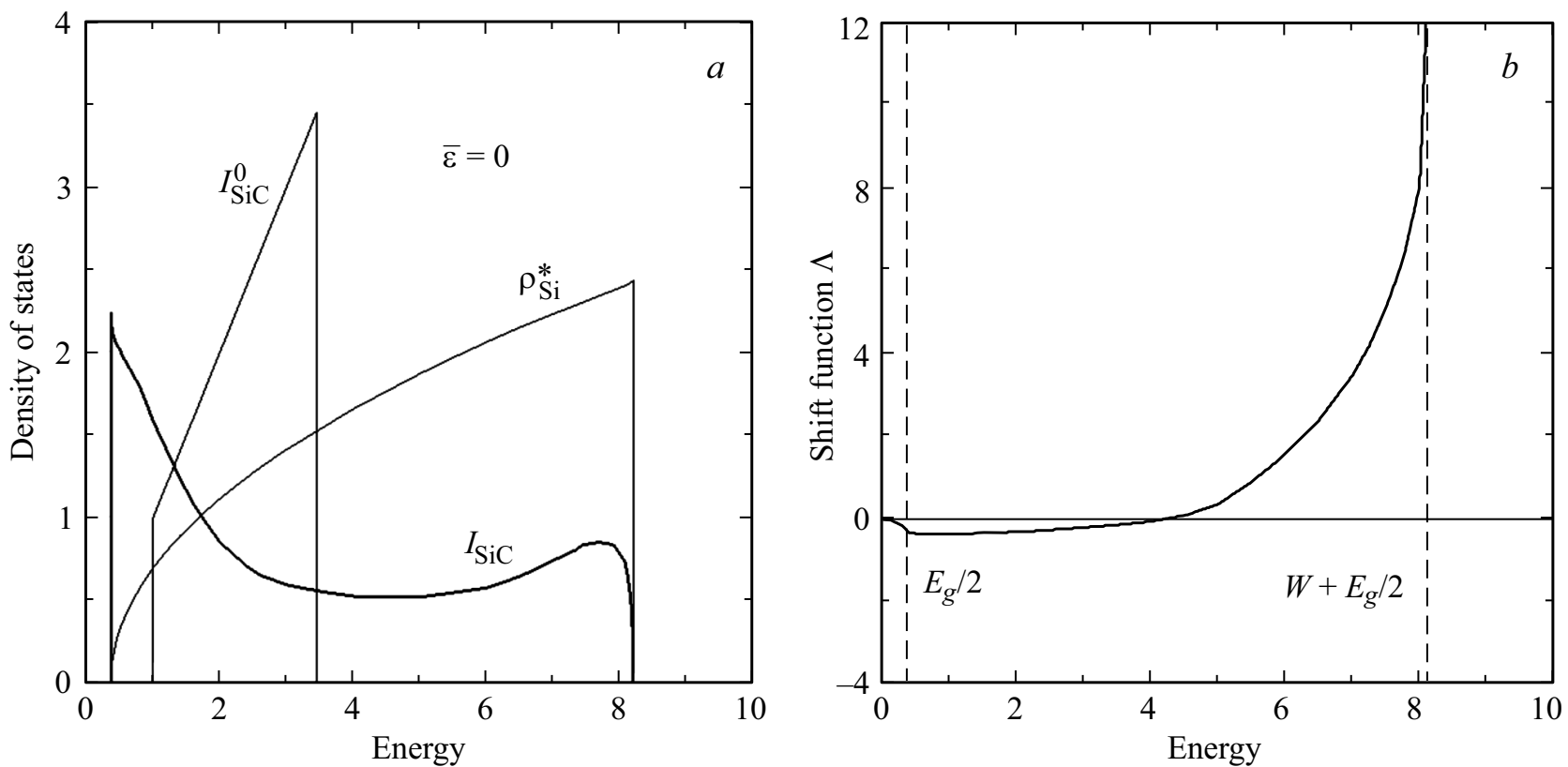

Рис. 1. $a-$ приведенная плотность состояний $I_{\mathrm{SiC}}(\omega)$ системы $2 \mathrm{D} \mathrm{SiC} / \mathrm{Si}$ при $\bar{\varepsilon}=0$. Тонкими линиями показыны приведенные плотности состояний не взаимодействующих между собой монослоя карбида кремния $I_{\mathrm{SiC}}^{0}(\omega)$ и кремниевой подложки $\rho_{\mathrm{Si}}^{*}(\omega) . b-$ функция сдвига $\Lambda(\omega)$. Энергия измеряется в ед. $t$, плотности состояний - в. ед. $(\pi t \sqrt{3})^{-1}$. На рисунке изображены только правые половины симметричных плотностей состояний и антисимметричной функции $\Lambda$.
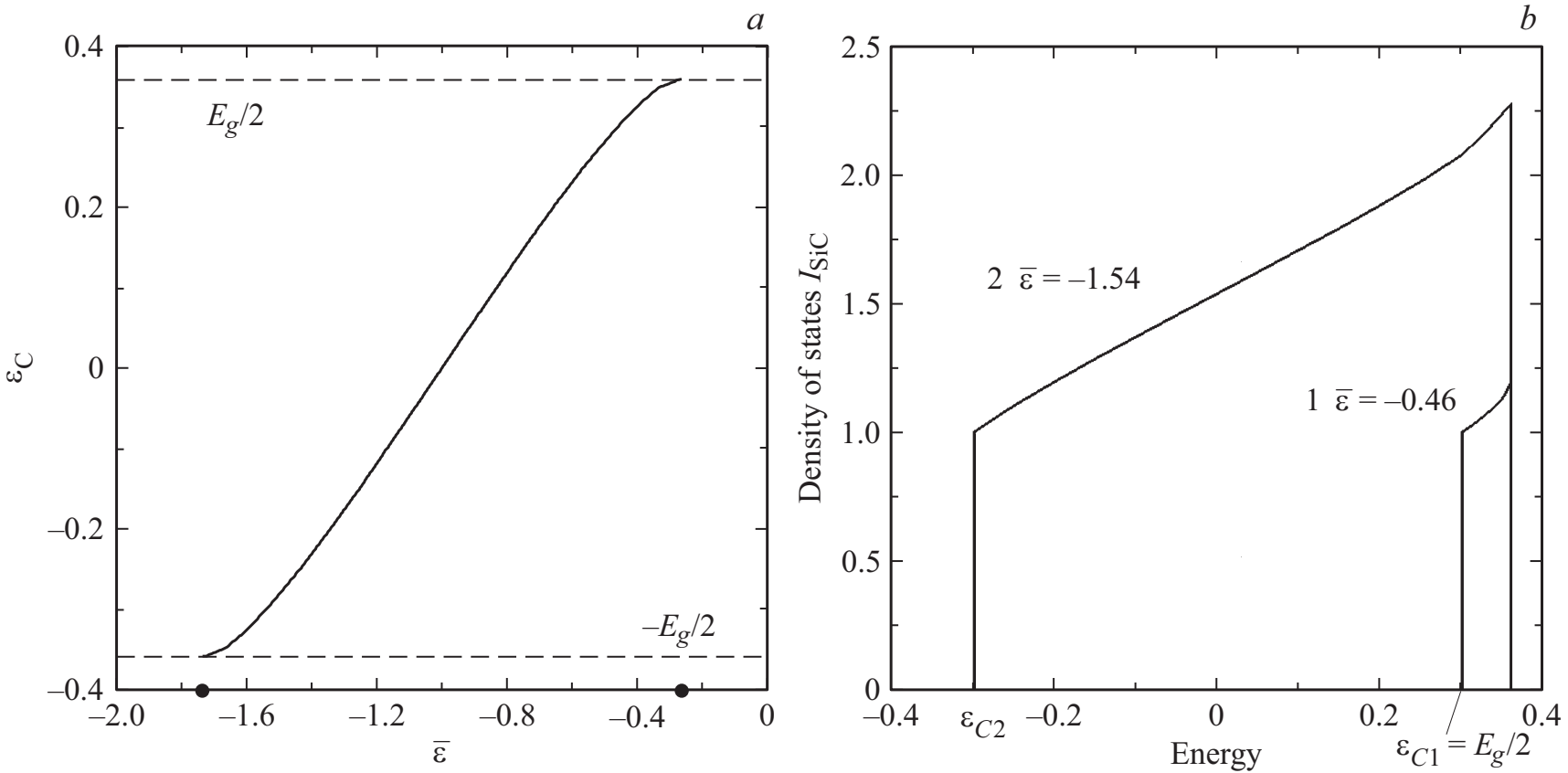

Рис. 2. $a$ - энергия дна зоны проводимости $\varepsilon_{C}$ в функции от положения центра запрещенной зоны $\bar{\varepsilon}$ для $2 \mathrm{D} \mathrm{SiC}$ в области запрещенной зоны Si-подложки. $b-$ приведенные плотности состояний $I_{\mathrm{SiC}}(\omega)$ системы $2 \mathrm{D} \mathrm{SiC} / \mathrm{Si}$ в области запрещенной зоны $\mathrm{Si}$-подложки. Энергия измеряется в ед. $t$, плотности состояний - в. ед. $(\pi t \sqrt{3})^{-1}$.

Si-подложки (это значение определяется из уравнения $\left.\bar{\varepsilon}=\varepsilon_{C}-\Lambda\left(\varepsilon_{C}\right)-R\right)$. В интервале $-4.19 t<\bar{\varepsilon}<-2.71 t$ система $2 \mathrm{D} \mathrm{SiC} / \mathrm{Si}$ является полупроводниковой, так как запрещенная зона $\mathrm{Si}$-подложки лишь частично перекрывается сплошным спектром 2D $\mathrm{SiC}$. Полностью запрещенная зона системы 2D $\mathrm{SiC} / \mathrm{Si}$ открывается при $\bar{\varepsilon} \leq-4.19 t$. Легко сообразить, что сдвиг $\bar{\varepsilon}$ в область положительных энергий приводит к зеркальному процессу: перекрытие запрещенной зоны системы $2 \mathrm{D} \mathrm{SiC/Si} \mathrm{из-за}$ смещения валентной зоны 2D SiC вверх по шкале энер- 
гий начинается с ее нижнего края. Отметим, что при выбранном нами соотношении $V=t$ плотность состояний $2 \mathrm{D} \mathrm{SiC/Si} \mathrm{существенно} \mathrm{отличается} \mathrm{от} \mathrm{плотностей} \mathrm{состо-}$ яний свободного листа 2D SiC и $\mathrm{Si}-$ подложки (рис. 1, $a$ ).

Рассмотрим теперь, как изменяются плотности состояний зоны проводимости и валентной зоны $2 \mathrm{D} \mathrm{SiC} / \mathrm{Si}$ при вариации $\bar{\varepsilon}$. Для дна зоны проводимости и потолка валентной зоны имеем $I_{\mathrm{SiC}}\left( \pm E_{g} / 2\right)=\left|\widetilde{\Omega}\left(\omega \pm E_{g} / 2\right)\right|$. Учтем, что в области краев зон $\Lambda(\omega)<0$ при $\omega>E_{g} / 2$ и $\Lambda(\omega)>0$ при $\omega<-E_{g} / 2$ (см. рис. $\left.1, b\right)$. Отсюда следует, что при $\bar{\varepsilon}<0$ плотность состояний $I_{\mathrm{SiC}}\left(E_{g} / 2\right)$ линейно увеличивается. При $\bar{\varepsilon}>0$ величина $I_{\mathrm{SiC}}\left(E_{g} / 2\right) \mathrm{c}$ ростом $\bar{\varepsilon}$ линейно уменьшается и обращается в нуль при $\bar{\varepsilon}=E_{g} / 2-\Lambda\left(E_{g} / 2\right)$. Дальнейшее увеличение $\bar{\varepsilon}$ ведет к линейному росту $I_{\mathrm{SiC}}\left(E_{g} / 2\right)$. Аналогичным образом ведет себя плотность состояний $I_{\mathrm{SiC}}\left(-E_{g} / 2\right)$ при условии замены $\bar{\varepsilon}$ на $-\bar{\varepsilon}$. В областях потолка зоны проводимости и дна валентной зоны имеем соответственно $\Lambda(\omega) \rightarrow \pm \infty$ (см. рис. $1, b)$ и $\widetilde{\Omega} \rightarrow \pm \infty$. При $\bar{\varepsilon}<0$ максимум функции $I_{\mathrm{SiC}}(\omega)$, лежащий вблизи потолка зоны проводимости, смещается вправо по шкале энергий, при $\bar{\varepsilon}<0-$ влево. Максимум функции $I_{\mathrm{SiC}}(\omega)$, лежащий вблизи потолка дна валентной зоны проводимости, ведет себя так же при условии замены $\bar{\varepsilon}$ на $-\bar{\varepsilon}$.

\section{3. Адсорбционная способность гетероструктуры 2D SiC/Si}

\section{1. Общие соотношения}

Рассмотрим адсорбцию на поверхности гетероструктуры 2D SiC/Si. В соответствии с общей теорией [23] плотность состояний $\rho_{a}(\omega)$ на адатоме имеет стандартный вид распределения Лоренца:

$$
\rho_{a}(\omega)=\frac{1}{\pi} \frac{\Gamma_{a}(\omega)}{\left(\omega-\varepsilon_{a}-\Lambda(\omega)\right)^{2}+\Gamma_{a}^{2}(\omega)} .
$$

Здесь $\varepsilon_{a}-$ уровень адатома, содержащий один электрон или одну дырку, $\Gamma_{a}(\omega)=\pi V_{a}^{2} \rho_{\mathrm{Sic}}(\omega)-$ функция уширения квазиуровня адатома $\left(V_{a}-\right.$ матричный элемент связи адатома с подложкой), функция сдвига $\Lambda_{a}(\omega)$ определяется формулой (4) с заменой $\Gamma(\omega)$ на $\Gamma_{a}(\omega)$. $\mathrm{C}$ целью получения аналитических результатов, аппроксимируем плотность состояний $\rho_{\mathrm{SiC}}(\omega)$ при $\bar{\varepsilon}(\omega)=0$ (рис. 1) функцией

$\rho_{\mathrm{SiC}}(\omega)=\left\{\begin{array}{l}\bar{\rho}_{\mathrm{SiC}}, \quad 1 \leq 2 \omega / E_{g} \leq 2 W / E_{g}+1 \\ \bar{\rho}_{\mathrm{SiC}}, \quad-1-2 W / E_{g} \leq 2 \omega / E_{g} \leq-1 \\ 0, \quad\left|2 \omega / E_{g}\right| \leq 1, \quad\left|2 \omega / E_{g}\right|>1+2 W / E_{g},\end{array}\right.$

где $\bar{\rho}_{\mathrm{SiC}}=W^{-1} \int_{E_{g} / 2}^{W+E_{g} / 2} \rho_{\mathrm{SiC}}(\omega) d \omega=$ const. Далее, полагая для простоты $2 W / E_{g} \gg 1$, приходим к модели
Халдейна-Андерсона $[23,24]$. Тогда из (8) получаем

$$
\Lambda_{a}(\omega)=\frac{\Gamma_{a}}{\pi} \ln \left|\frac{\omega-E_{g} / 2}{\omega+E_{g} / 2}\right|,
$$

где $\Gamma_{a}=\pi V_{a}^{2} \bar{\rho}_{\mathrm{SiC}}$.

Число заполнения адатома $n_{a}$, равное при нулевой температуре $n_{a}=\int_{-\infty}^{E_{\mathrm{F}}} \rho_{a}(\omega) d \omega\left(E_{\mathrm{F}}-\right.$ уровень Ферми $)$, удобно представить в виде суммы вклада $n_{v}$ валентной зоны и вклада $n_{l}$ локального уровня с энергией $\omega_{l}$ при условии, что $\omega_{l}<E_{\mathrm{F}}$. В [23] показано, что первый из этих вкладов равен

$$
n_{v}=\frac{1}{\pi} \operatorname{arcctg} \frac{\varepsilon_{a}+R}{\Gamma_{a}}, R=\frac{E_{g}}{4} \sqrt{1+\frac{8 \Gamma_{a}}{\pi E_{g}}} .
$$

Положение локального уровня $\omega_{l}$ определяется из решения уравнения

$$
\omega-\varepsilon_{a}-\Lambda_{a}(\omega)=0
$$

в области $|\omega| \leq E_{g} / 2[23,24]$. Число заполнения локального состояния равно

$$
n_{l}=\left(1+\frac{\Gamma_{a}}{2 \pi} \frac{E_{g}}{\left(E_{g} / 2\right)^{2}-\omega_{l}^{2}}\right)^{-1} \Theta\left(E_{\mathrm{F}}-\omega_{l}\right),
$$

где $\Theta\left(E_{\mathrm{F}}-\omega_{l}\right)-$ функция Хэвисайда.

\section{2. Адсорбция атомов I и VII групп на поверхности 2D SiC/Si}

Начнем с оценок вклада валентной зоны в число заполнения $n_{v}$. Энергия одноэлектронного уровня адатома щелочного металла равна $\varepsilon_{a}=-I+e^{2} / d$ $+\left(\chi+E_{g} / 2\right)$ [24], для однодырочного уровня галогена имеем $\varepsilon_{a}=-A-e^{2} / d+\left(\chi+E_{g} / 2\right)$ [24], где $I-$ энергия ионизации [25], $A-$ сродство атома к электрону [25], $d$ - длина адсорбционной связи, $e-$ величина заряда электрона. Рассмотрим тип $A$ (atop) адсорбции, когда адатом связан непосредственно только с одним поверхностным атомом С или $\mathrm{Si}$. Полагаем $d(X-\mathrm{C}, \mathrm{Si})=r_{a}(\mathrm{C}, \mathrm{Si})+r_{a}(X), \quad$ где $r_{a}(\mathrm{C}, \mathrm{Si})-$ атомные радиусы углерода или кремния при адсорбции на С- или $\mathrm{Si}$-грани $\mathrm{SiC}, r_{a}(X)$ - атомный радиус щелочного металла $\mathrm{M}$ или галогена $\mathrm{H}$ (величины радиусов брались для М из [25], для Н (по Полингу) - из [26]). Для адсорбции атома лития положим $\Gamma_{a}=1$ эВ. Значения $\Gamma_{a}$ для адатомов других щелочных металлов определим исходя из того, что матричный элемент $V \propto d^{-2}$ и $\Gamma_{a} \propto V^{2}$. Тогда $\Gamma_{a}(M)=\gamma \Gamma_{a}(\mathrm{Li})$, где $\gamma=[d(\mathrm{Li}-\mathrm{C}, \mathrm{Si}) / d(\mathrm{M}-\mathrm{C}, \mathrm{Si})]^{4}$. В случае адсорбции галогенов нужно дополнительно учесть, что в соответствии с теорией Харрисона $V_{\mathrm{H}}=\left(V_{s p \sigma}+\sqrt{3} V_{p p \sigma}\right) / 2$, тогда, как в случае адсорбции щелочных металлов, $V_{\mathrm{M}}=\left(V_{s s \sigma}+\sqrt{3} V_{s p \sigma}\right) / 2$ [27]. Тогда $\Gamma_{a}(\mathrm{H})=\gamma^{\prime} \gamma \Gamma_{a}(\mathrm{Li})$, 
Таблица 1. Значения длин адсорбционной связей $d(\AA)$, энергии $\varepsilon_{a}$ и полуширины $\Gamma_{a}$ квазиуровня, энергий локальных уровней $\omega_{l}$, ионных $E_{\text {ion }}$ и металлических $E_{\text {met }}$ вкладов в энергию адсорбции (эВ), вкладов валентной зоны $n_{v}$ и локального уровня $n_{l}$ в числа заполнения и значений заряда $Z$ при адсорбции щелочных металлов на поверхностных атомах $\mathrm{C}$ и $\mathrm{Si}$ гетероструктуры 2D $\mathrm{SiC} / \mathrm{Si}$

\begin{tabular}{c|c|c|c|c|c}
\hline Адсорбат & $\mathrm{Li}$ & $\mathrm{Na}$ & $\mathrm{K}$ & $\mathrm{Rb}$ & $\mathrm{Cs}$ \\
\hline Атом C & & & & & \\
$d$ & 2.34 & 2.63 & 3.13 & 3.25 & 3.30 \\
$\varepsilon_{a}$ & 0.70 & 0.78 & 1.36 & 1.48 & 1.72 \\
$\Gamma_{a}$ & 1 & 0.63 & 0.31 & 0.27 & 0.23 \\
$n_{\nu}$ & 0.19 & 0.13 & 0.05 & 0.04 & 0.03 \\
$\omega_{l}$ & 0.31 & 0.41 & 0.56 & 0.56 & 0.56 \\
$n_{l}$ & 0.37 & 0.39 & 0 & 0 & 0 \\
$Z^{<}$ & 0.81 & 0.87 & 0.95 & 0.96 & 0.97 \\
$Z^{>}$ & 0.44 & 0.48 & 0.95 & 0.96 & 0.97 \\
$-E_{\text {ion }}^{<}$ & 1.01 & 1.04 & 1.04 & 1.02 & 1.03 \\
$-E_{\text {ion }}^{>}$ & 0.30 & 0.32 & 1.02 & 1.00 & 0.98 \\
$-E_{\text {met }}$ & 0.77 & 0.55 & 0.34 & 0.30 & 0.26 \\
\hline Атом Si & & & & & \\
$d$ & 2.75 & 3.04 & 3.54 & 3.66 & 3.80 \\
$\varepsilon_{a}$ & 0.47 & 0.59 & 1.23 & 1.35 & 1.61 \\
$\Gamma_{a}$ & 0.52 & 0.35 & 0.19 & 0.17 & 0.14 \\
$n_{v}$ & 0.13 & 0.09 & 0.03 & 0.03 & 0.02 \\
$\omega_{l}$ & 0.28 & 0.40 & 0.56 & 0.56 & 0.56 \\
$n_{l}$ & 0.56 & 0.54 & 0 & 0 & 0 \\
$Z^{<}$ & 0.83 & 0.91 & 0.97 & 0.97 & 0.98 \\
$Z^{>}$ & 0.10 & 0.37 & 0.97 & 0.97 & 0.98 \\
$-E_{\text {ion }}^{<}$ & 0.99 & 0.98 & 0.95 & 0.93 & 0.91 \\
$-E_{\text {ion }}^{>}$ & 0.13 & 0.16 & 0.95 & 0.93 & 0.91 \\
$-E_{\text {met }}$ & 0.68 & 0.52 & 0.34 & 0.31 & 0.28 \\
& & & & &
\end{tabular}

Примечание. $Z^{<(>)}$означает, что уровень Ферми $E_{\mathrm{F}}$ лежит ниже (выше) локального уровня $\omega_{l}$.

где $\gamma^{\prime}=0.97$ (здесь мы считаем все матричные элементы $V_{l l^{\prime} \sigma}$ положительными величинами, см. подробнее $[24,27])$. Значения $\varepsilon_{a}, \Gamma_{a}$ и $n_{v}$ приведены в табл. 1 и 2.

Перейдем теперь к оценкам вкладов $n_{l}$ локальных уровней $\omega_{l}$. Рис. 3 иллюстрирует решение уравнения (11) в области запрещенной зоны $|\omega| \leq E_{g} / 2$ системы (напомним, что при $|\bar{\varepsilon}| \leq 0.26 t$ запрещенная зона гетероструктуры 2D $\mathrm{SiC} / \mathrm{Si}$ пуста). Значения $\omega_{l}$ и $n_{l}$ сведены в табл. 1 и 2.

Обсудим переход заряда при адсорбции щелочных металлов (табл. 1). Убыль вклада валентной зоны $n_{v}$ в ряду $\mathrm{Li} \rightarrow \mathrm{Cs}$ обусловлена смещением положительного уровня адатома $\varepsilon_{a}$ в область положительных энергий (из-за уменьшения потенциала ионизации $I$ в том же ряду) и сужением квазиуровня адатома $\Gamma_{a}$ вследствие роста длины адсорбционной связи $d$ (см. (10)). Та же тенденция наблюдается и для вклада локальных состояний $n_{l}$ : рост $\varepsilon_{a}$ смещает изображенную на рис. 3 прямую линию вправо, а уменьшение $\Gamma_{a}$ увеличивает наклон этой прямой. Сказанное относится как к адсорбции на атомах C, так и к адсорбции на атомах $\mathrm{Si}$. Нулевые зна-
Таблица 2. То же, что и в табл. 1, но для адсорбции галогенов

\begin{tabular}{c|r|r|r|r}
\hline Адсорбат & \multicolumn{1}{|c|}{$\mathrm{F}$} & \multicolumn{1}{c|}{$\mathrm{Cl}$} & \multicolumn{1}{c}{$\mathrm{Br}$} & \multicolumn{1}{c}{$\mathrm{I}$} \\
\hline Атом C & & & & \\
$d$ & 1.41 & 1.76 & 1.91 & 2.10 \\
$\varepsilon_{a}$ & -1.40 & -1.12 & -0.70 & -0.22 \\
$\Gamma_{a}$ & 7.36 & 3.03 & 2.19 & 1.50 \\
$n_{v}$ & 0.43 & 0.49 & 0.45 & 0.36 \\
$\omega_{l}$ & -0.15 & -0.11 & -0.19 & -0.08 \\
$n_{l}$ & 0.10 & 0.22 & 0.26 & 0.36 \\
$Z^{<}$ & -0.43 & -0.49 & -0.45 & -0.36 \\
$Z^{>}$ & -0.53 & -0.71 & -0.71 & -0.72 \\
$-E_{\text {ion }}^{<}$ & 0.47 & 0.49 & 0.38 & 0.22 \\
$-E_{\text {ion }}^{>}$ & 0.83 & 1.03 & 0.95 & 0.89 \\
$-E_{\text {met }}$ & 3.04 & 1.68 & 1.34 & 1.03 \\
\hline Атом Si & & & & \\
$d$ & 1.82 & 2.17 & 2.32 & 2.51 \\
$\varepsilon_{a}$ & -0.83 & -0.73 & -0.37 & 0.06 \\
$\Gamma_{a}$ & 2.65 & 1.31 & 1.00 & 0.73 \\
$n_{v}$ & 0.47 & 0.46 & 0.37 & 0.23 \\
$\omega_{l}$ & -0.29 & -0.28 & -0.17 & -0.03 \\
$n_{l}$ & 0.19 & 0.33 & 0.44 & 0.54 \\
$Z^{<}$ & -0.47 & -0.46 & -0.37 & -0.23 \\
$Z^{>}$ & -0.66 & -0.79 & -0.81 & -0.77 \\
$-E_{\text {ion }}^{<}$ & 0.43 & 0.36 & 0.21 & 0.08 \\
$-E_{\text {ion }}^{>}$ & 0.87 & 1.04 & 1.02 & 0.85 \\
$-E_{\text {met }}$ & 2.02 & 1.28 & 1.07 & 0.87 \\
& & & &
\end{tabular}

чения $n_{l}$ при адсорбции $\mathrm{K}, \mathrm{Rb}$ и $\mathrm{CS}$ связаны с тем обстоятельством, что соответствующие локальные уровни $\omega_{l}$ перекрываются с дном зоны проводимости (см. (12)).

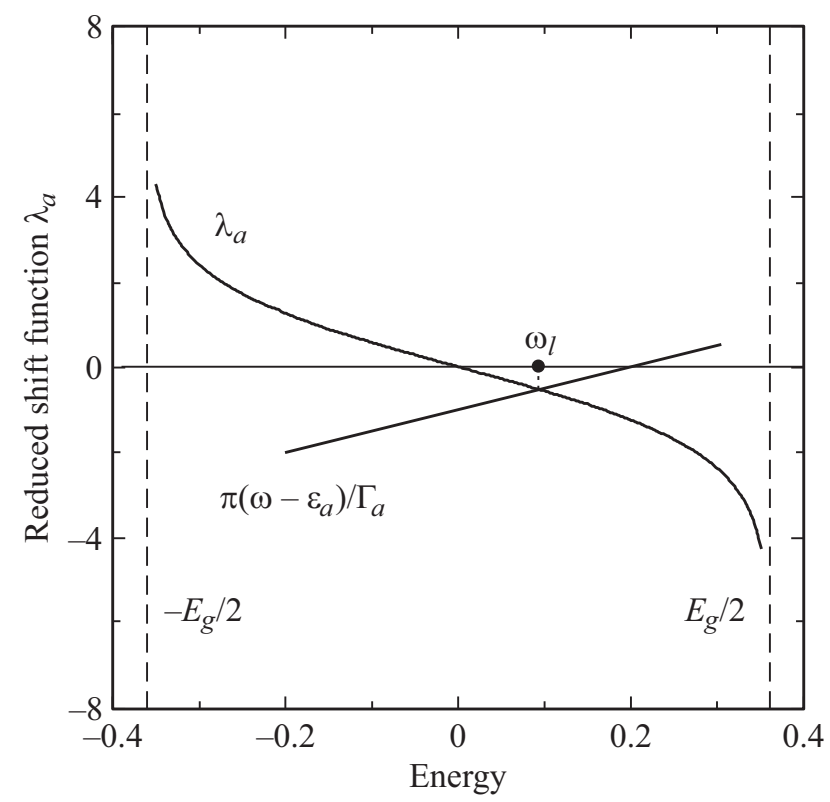

Рис. 3. К решению уравнения (11) в области запрещенной зоны $|\omega| \leq E_{g} / 2$. Точка пересечения прямой линии $\pi\left(\omega-\varepsilon_{a}\right) / \Gamma_{a}$ с приведенной плотностью состояний $\lambda_{a}(\omega)=\ln \left|\left(E_{g} / 2-\omega\right) /\left(E_{g} / 2+\omega\right)\right|$ определяет положение локального уровня $\omega_{l}$. 
Аналогичная в целом картина имеет место и для адсорбции галогенов: тенденции в ряду $\mathrm{F} \rightarrow \mathrm{I}$ те же самые. Отметим, что в случае адсорбции $\mathrm{Br}$ и I на атомах кремния имеем соответственно $n=1.09$ и 1.01 . Малая ошибка возникает из-за приближенного характера выражения (10).

Перейдем к оценкам энергии адсорбции. Ионная составляющая энергии адсорбции может быть вычислена по электростатической формуле

$$
E_{\mathrm{ion}}=\frac{(Z e)^{2}}{4 d}
$$

где заряд адатома $Z=1-n$ при адсорбции щелочных металлов и $Z=-n$ при адсорбции галогенов. Значения ионной составляющей энергии адсорбции приведены в табл. 1 и 2. При этом большое значение имеет тип проводимости подложки. Если предположить, что уровень Ферми расположен вблизи дна зоны проводимости (n-тип) и все локальные уровни заполнены, то получаем заряд $Z^{>}$, минимальный для адатомов щелочных металлов и максимальный по модулю для адатомов галогенов. Если же уровень Ферми лежит вблизи потолка валентной зоны ( $p$-тип), то заряд $Z^{<}$щелочных металлов максимален, а заряд галогенов минимален по модулю (в случае адсорбции $\mathrm{Br}$ и I на атомах кремния заряды $Z^{>}$приняты равными -1$)$. Следует отметить, что значения $\left|E_{\text {ion }}\right|$ для адсорбции щелочных атомов и галогенов на поверхностных атомах углерода выше, чем для адсорбции на поверхностных атомах кремния.

Металлическую составляющую энергии адсорбции $E_{\text {met }}$ оцениваем исходя из соотношения неопределенностей $\Delta x \cdot \Delta p \sim \hbar$. Полагая, что в изолированном атоме $\Delta x \sim r_{a}$, а в адсорбированном состоянии $\Delta x \sim d$, получим выигрыш в кинетической энергии $\Delta E_{\mathrm{kin}} \sim\left(\hbar^{2} / 2 m d^{2}\right)\left[1-\left(r_{a} / d\right)^{2}\right]$, где $m-$ масса свободного электрона. Этот выигрыш в кинетической энергии, связанный с делокализацией электрона при адсорбции, принимаем за оценку металлической составляющей энергии адсорбции:

$$
E_{\mathrm{met}}=-\frac{\hbar^{2}}{2 m d^{2}}\left(1-\frac{r_{a}^{2}}{d^{2}}\right) .
$$

Результаты оценок приведены в табл. 1 и 2. Как следует из таблиц, величина металлического вклада уменьшается в рядах $\mathrm{Li} \rightarrow \mathrm{Cs}$ и $\mathrm{F} \rightarrow \mathrm{I}$, что обусловлено соответствующим ростом длин адсорбционной связи $d$. Значениями $d$ объясняется также и то обстоятельство, что $\left|E_{\text {met }}\right|$ для адсорбции галогенов значительно выше, чем для адсорбции щелочных металлов. То же неравенство имеет место при сравнении $\left|E_{\text {met }}\right|$ для адсорбции на атомах С и $\mathrm{Si}$.

До сих пор мы рассматривали случай промежуточной связи 2D $\mathrm{SiC}$ с Si-подложкой, положив $V=t$. В пределе слабой связи $V / t \ll 1$ в нулевом приближении можно считать, что атом адсорбируется на поверхности 2D $\mathrm{SiC}$. Тогда плотность состояний на адатоме дается выражением (7) с заменой $\Gamma_{a}(\omega)$ на $\Gamma_{a}^{0}(\omega)=\pi V^{2} \rho_{\mathrm{SiC}}^{0}(\omega)$, где плотность состояний свободного графена $\rho_{\mathrm{SiC}}^{0}(\omega)$ определяется формулой $(2)$ с заменой $\widetilde{\Omega}$ на $\Omega$. Определив функцию сдвига $\Lambda_{a}^{0}(\omega)$ из выражения (4), можно приступать к расчетам чисел заполнения и ионной составляющей энергии адсорбции. Подобная задача рассматривалась в работе [28].

В нулевом приближении предела сильной связи $V / t \gg 1$ атом $\mathrm{C}(\mathrm{Si})$ монослоя $\mathrm{SiC}$ можно считать изолированным адатомом на $\mathrm{Si}$-подложке, которому соответствует функция Грина $g_{\mathrm{C}(\mathrm{Si})}^{-1}=\omega-\varepsilon_{\mathrm{C}(\mathrm{Si})}-\Lambda(\omega)+i \Gamma(\omega)$, где $\Gamma(\omega)=\pi V^{2} \rho_{\mathrm{AB}}(\omega), \rho_{\mathrm{AB}}(\omega)$ дается формулой (3), $\Lambda(\omega)$ - формулой (4). Вводя функцию Грина адсорбируемой частицы $g_{a}^{-1}(\omega)=\omega-\varepsilon_{a}+i 0^{+}$, с помощью уравнения Дайсона [24] получаем функцию Грина частицы, связанной с адатомом $\mathrm{C}(\mathrm{Si})$ :

$$
\widetilde{g}_{a}^{\mathrm{C}(\mathrm{Si})}=\frac{g_{a}}{1-g_{a} g_{\mathrm{C}(\mathrm{Si})} V_{a}^{2}} .
$$

Плотность состояний $\widetilde{\rho}_{a}^{\mathrm{C}(\mathrm{Si})}=-\pi^{-1} \operatorname{Im} \widetilde{g}_{a}^{\mathrm{C}(\mathrm{Si})}$ позволяет вычислить соответствующее число заполнения и ионные вклады в энергию адсорбции. Отметим, что режим связи между 2D $\mathrm{SiC}$ и $\mathrm{Si}$-подложкой во многом зависит от технологии формирования системы 2D $\mathrm{SiC} / \mathrm{Si}$.

Кратко обсудим модельное описание адсорбции при $\bar{\varepsilon} \neq 0$. Предположим, что ширина запрещенной зоны системы равна $E_{g}^{\prime}$. Тогда вместо (8) можно ввести плотность состояний вида:

$$
\rho_{\mathrm{SiC}}^{\prime}(\omega)=\left\{\begin{array}{c}
D_{ \pm} \mp\left[\left(\omega+W+E_{g}^{\prime} / 2\right) / W\right] D_{\mp}, \\
-W-E_{g}^{\prime} / 2 \leq \omega \leq-E_{g}^{\prime} / 2, \\
D_{ \pm} \mp\left[\left(\omega-W-E_{g}^{\prime} / 2\right) / W\right] D_{\mp}, \\
W+E_{g}^{\prime} / 2 \geq \omega \geq E_{g}^{\prime} / 2, \\
0, \quad|\omega|<E_{g}^{\prime} / 2 .
\end{array}\right.
$$

Здесь $D_{ \pm}=\rho_{\mathrm{SiC}}\left( \pm E_{g}^{\prime} / 2\right)$, верхний (нижний) знак между слагаемыми соответствует случаю $\bar{\varepsilon}<0(\bar{\varepsilon}>0)$, начало отсчета энергии совмещено с центром запрещенной зоны $E_{g}^{\prime}$. Выражение (15) позволяет найти соответствующую функцию сдвига $\Lambda_{a}^{\prime}(\omega)$. В случае $E_{g}^{\prime}=0$ имеем задачу об адсорбции на металле, где модели плотностей состояний значительно проще $[23,24]$. Отметим, что мы вынуждены варьировать значение параметра $\bar{\varepsilon}$, так как какие-либо сведения относительно электронного сродства 2D $\mathrm{SiC}$, насколько известно авторам, отсутствуют.

\section{4. Заключение}

В настоящей работе мы рассмотрели адсорбцию атомов I и VII групп, являющихся наиболее электроположительными и электроотрицательными элементами соответственно. Первые из них выступают как доноры и понижают работу выхода адсорбционной системы, вторые являются акцепторами и работу выхода адсорбционной системы повышают. Отметим, что адсорбция является одним из эффективных способов допирования двумерных структур. 
Здесь мы считали температуру равной нулю. Необходимо отметить, что вклад валентной зоны $n_{v}$ в суммарное число заполнения крайне слабо зависит от температуры [23]; температура, однако, может оказать существенное влияние на заполнение локальных уровней $n_{l}$ [29]. В настоящей работе мы ограничились случаем адсорбции одного атома. Не сложно, однако, обобщить полученные результаты на конечные степени покрытия [24].

\section{Благодарности}

Авторы признательны С.А. Кукушкину за предложение темы и полезные обсуждения.

\section{Конфликт интересов}

Авторы заявляют об отсутствии конфликта интересов.

\section{Список литературы}

[1] A.K. Geim, I.V. Grigorieva. Nature, 499, 419 (2013).

[2] G. Mukhopadhyay, H. Behera. World J. Engin., 10, 39 (2013).

[3] H.L. Zhuang, A.K. Singh, R.G. Hennig. Phys. Rev. B, 87, 165415 (2013).

[4] C.-J. Tong, H. Zhang, Y.-N. Zhang, H. Liu, L.-M. Liu. J. Mater. Chem. A, 2, 17971 (2014).

[5] И.В. Антонова. ФТП, 50, 67 (2016).

[6] A. Onen, D. Kecik, E. Durgun, S. Ciraci. Phys. Rev. B, 93, 085431 (2016).

[7] R. Roldán, L. Chirolli, E. Prada, J.A. Silva-Guillén, P. San Josea, F. Guinea. Chem. Soc. Rev., 46, 4387 (2017).

[8] D. Kecik, A. Onen, M. Konuk, E. Gürbüz, F. Ersan, S. Cahangirov, E. Akürk, E. Durgun, S. Ciraci. Appl. Phys. Rev., 5, 011105 (2018).

[9] С.Ю. Давыдов. ФТП, 54 (5), 446 (2020).

[10] С.А. Кукушкин, А.В. Осипов, Н.А. Феоктистов. ФТТ, 56, 1457 (2014).

[11] S.A. Kukushkin, A.V. Osipov. J. Phys. D: Appl. Phys., 47, 513001 (2014).

[12] Г.В. Бенеманская, П.А. Дементьев, С.А. Кукушкин, А.В. Осипов, С.Н. Тимошнев. Письма ЖТФ, 45 (5), 17 (2019).

[13] С.Ю. Давыдов. ФТТ, 58, 779 (2016).

[14] С.Ю. Давыдов. ФТТ, 58, 1182 (2016).

[15] S.Yu. Davydov. In: Handbook of Graphene, V. 2: Physics, Chemistry, and Biology, ed. by T. Stauber (N.Y., Scrivener Publishing LLC, 2019) chap. 17, p.549.

[16] С.Ю. Давыдов. ЖТФ, 84 (4), 155 (2014).

[17] Ф. Бехштедт, Р. Эндерлайн. Поверхности и границы раздела полупроводников (М., Мир, 1990).

[18] С.Ю. Давыдов. ФТП, 53, 706 (2019).

[19] У. Харрисон. Электронная структура и свойства твердых тел (М., Мир, 1983).

[20] W.A. Harrison. Phys. Rev. B, 31, 2121 (1985).

[21] W.A. Harrison. Phys. Rev. B, 27, 3592 (1983).

[22] С.Ю. Давыдов. ФТП, 48, 49 (2014).

[23] С.Ю. Давыдов, С.В. Трошин. ФТТ, 49, 1508 (2007).
[24] С.Ю. Давыдов. Теория адсорбиии: метод модельных гамильтонианов (СПб., Изд-во СПбГЭТУ „ЛЭТИ“, 2013). Электронный адрес: twirpx.com/file/1596114/

[25] Физические величины. Справочник, под ред. Е.С. Григорьева, Е.3. Мейлихова (М., Энергоатомиздат, 1991).

[26] Краткий справочник физико-химических величин, под ред. К.П. Мищенко, А.А. Равделя (Л., Химия, 1974).

[27] С.Ю. Давыдов, Г.И. Сабирова. Письма ЖТФ, 36, (24), 77 (2010).

[28] С.Ю. Давыдов. ФТП, 51, 226 (2017).

[29] С.Ю. Давыдов. ЖТФ, 86 (7), 145 (2016).

Редактор Г.А. Оганесян

\section{Heterostructure 2D SiC/Si: electron states and adsorptive capability}

\author{
S.Yu. Davydov' ${ }^{1}$, A.V. Zubov ${ }^{2}$ \\ ${ }^{1}$ loffe Institute, \\ 194021 St. Petersburg, Russia \\ ${ }^{2}$ St. Petersburg National University \\ of Information Technology, Mechanics and Optics \\ (ITMO University), \\ 197101 St. Petersburg, Russia
}

\begin{abstract}
Model for heterostructure, composed of the silicon carbide monolayer, fabricated on the bulk silicon substrate, is proposed. The problem on the atoms of alkali metals and halogens adsorption on the 2D $\mathrm{SiC} / \mathrm{Si}$ carbon and silicon surface atoms is considered. Analytical estimates for the charge transfer and adsorption energy are given.
\end{abstract}

